\title{
Space Charge Formation and its Modified Electric Field under Applied Voltage Reversal and Temperature Gradient in XLPE Cable
}

\author{
M. Fu and L. A. Dissado \\ Department of Engineering \\ University of Leicester, Leicester, LE1 7RH, UK \\ G. Chen \\ School of Electronics and Computer Science \\ University of Southampton \\ Southampton, SO17 1BJ, UK \\ J. C. Fothergill \\ Department of Engineering \\ University of Leicester, Leicester, LE1 7RH, UK
}

\begin{abstract}
The results of space charge evolution in cross-linked polyethylene power cables under dc electrical field at a uniform temperature and during external voltage polarity reversal are presented in the paper. A mirror image charge distribution was observed in the steady state, but the pre-existing field altered the way in which the steady state charge distribution was formed from that obtaining when the cable was first polarized. Polarity reversing charge was generated in the middle of the insulation and moved towards the appropriate electrodes under the influence of a field in excess of the maximum applied field. Our results show that the mirror effect is a steady state effect that is due to crossinterface currents that depend only on the interface field and not its polarity. Measurements on cable sections with an elevated mean temperature and temperature gradient show that the interface currents are temperature dependent, and that differences between the activation energies of the interface and bulk currents can eliminate and possibly even invert the polarity of the space charge distribution.
\end{abstract}

Index Terms - Space charge, PEA, XLPE insulated power cables, voltage polarity reversal, temperature gradient, "mirror image effect".

\section{INTRODUCTION}

THE renewed interest in high voltage direct current (HVDC) transmission has led to many manufacturers worldwide investing in polymer insulated dc power cables. Cross-linked polyethylene (XLPE) is the most common form of polymeric insulation, however the features that make it a good insulator, may paradoxically lead to problems when it is used in de operation. Thus its low carrier mobility and high trapping rates may, under certain conditions, give rise to space charge in the body of the insulating material. This will result in localised electric stress enhancement and even premature failure of the cable insulation when the localised fields exceed the design values [1-3]. This issue has stimulated the investigation of space charge accumulation and retention, and abundant data has been obtained from film and plaque

Manuscript received on 3 November 2006, in final form 18 January 2008. samples over the past two decades [4-11]. However, less attention has been paid to space charge dynamics in full sized cables presumably due to a limited range of experimental systems suitable for examining cables. Such investigations would however be more relevant to practical situations as all the features specific to cable design, such as insulator/semiconducting interfaces, insulating material processing, and divergent electric field would be fully reflected in the space charge behaviour. They would also make it possible to investigate the effect of a temperature gradient in radial insulation on the space charge accumulation, and hence replicate the conditions experienced when the cable is loaded in service.

In a dc transmission system, bi-directional power flow may be achieved by exchanging the roles of rectifier (sending end) and inverter (receiving end), i.e. through voltage polarity reversal. The presence of space charge may result in an enhanced electric field within the insulation as its spatial 
distribution may not be able to synchronously follow the polarity reversal due to low charge carrier mobility, but the magnitude and the location is unpredictable. This could pose a vital threat to the cable insulation in service. The temperature gradient existing across the insulation in a fully loaded cable will make the space charge distribution, and consequently the electric field more complex. The investigation of space charge at elevated temperature in cable geometry is therefore of importance for the electric field distribution and performance of dc cable insulation.

Here we present results for the space charge evolution and response to external voltage polarity reversal measured on full size XLPE power cables using the pulsed electro-acoustic (PEA) technique. The corresponding electric field distributions along the radial direction are derived from the space charge distribution, and the local field distribution is discussed in terms of the evolution of mirror space charge distributions in the steady state, and the basic physics behind the formation of an inverted space charge distribution on reversing the polarity of the conductor potential. The effect of a temperature gradient on space charge in cable insulation is also investigated, and it is argued that the reduction of space charge observed is caused by differences in the temperature dependence of the bulk conductivity and those applying to the cross-interface currents.

\section{EXPERIMENTAL}

\subsection{PEA FOR CABLE SAMPLES}

The PEA system used for space charge distribution in cable insulation measurement is schematically shown in figure 1. A detailed description of the principle of PEA and the modified system suitable for cable geometry has been given elsewhere $[1,12,13]$ and only a brief overview is given in this paper.

There have been several reports on the application of PEA for space charge measurement in full size cables [13-16] in most of which a curve-shaped ground electrode, transducer, and acoustic absorber block were designed to fit around the diameter of the cable sample. For different cable sizes, modification is required to the above components in addition to extra care in the sample assembly in order to ensure a good acoustic contact between the cable and curved electrode. The PEA system employed in this work adopts a flat ground electrode which enables the system to be easily applied to cables with different radii such as described in [17].

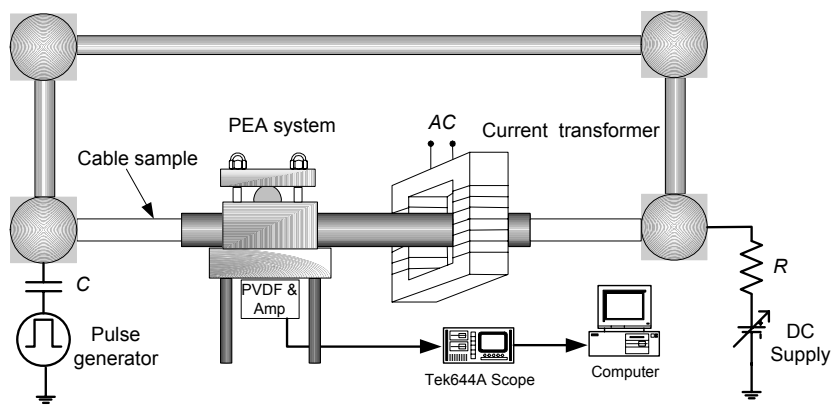

Figure 1. Schematic diagram of PEA system for cable geometry

\subsection{CABLE SAMPLES AND TEMPERATURE GRADIENT}

Two types of cable sample were tested in the research. One is a commercial XLPE ac power cable with an insulation thickness of $3.6 \mathrm{~mm}$, Cable A, which is tested to investigate the space charge response to the voltage polarity reversal. The other one is a prototype HVDC power cable with a $5.7 \mathrm{~mm}$ thick insulation. This was received in both degassed and undegassed condition, but only results from the un-degassed form (Cable B) are reported here. Due to its thicker insulation, the latter cable was the one chosen for the temperature gradient experiments. In order to allow sufficient clear distance from the voltage-applying terminal to the ground electrode the outer semiconductive screens at the two ends of the cable were stripped, and the remaining section used as the outer-earthed electrode. Stress relief rings were also built at the screen cuts to reduce the likelihood of flashover along the insulation surface.

A current transformer was set up in the testing rig, as shown in Figure 1, to generate a radial temperature distribution across the cable insulation by means of inductionheating (joule heating $I^{2} R$ ). It is assumed that the thermal conductivity of the cable insulation remains constant over the temperature range used in the present study (e.g. $30{ }^{\circ} \mathrm{C}$ to 80 ${ }^{\circ} \mathrm{C}$ ). For a cable sample with a conductor radius of $r_{c}$ and outer sheath radius of $r_{s}$, the temperature distribution across the insulation thickness can be calculated as a function of the radius $r$ [18] through:

$T(r)=T_{s}+\frac{T_{c}-T_{s}}{\ln \left(r_{s} / r_{c}\right)} \ln \left(\frac{r_{s}}{r}\right)$

where $T_{s}$ and $T_{c}$ are the temperature of the outer screen and the conductor respectively.

A typical induced ac current of 350 A was used to heat the cable, which has a cross section of $78 \mathrm{~mm}^{2}$ aluminium conductor and $5.7 \mathrm{~mm}$ thick insulation. A temperature of 70 ${ }^{\circ} \mathrm{C}$ at the conductor was obtained with a temperature difference of $13{ }^{\circ} \mathrm{C}$ across the insulation, when the outer semiconductor was kept at ambient by natural convection cooling.

\subsection{VOLTAGE REVERSAL EXPERIMENTAL PROCEDURES}

In the voltage reversal test the sample was initially stressed with a positive voltage at the centre conductor and the space charge distribution was measured at different stressing times. When the space charge distribution appeared to reach a steady state the external de voltage was switched off and the cable was short-circuited for a short time to release the surface static charge. Then the dc voltage was switched to the opposite polarity and ramped up again. In order to make the voltage reversal a more realistic representation of the operation of $\mathrm{dc}$ transmission systems, the above implementation was finished within about $90 \mathrm{~s}$. The space charge distribution measurement was then conducted over the following stressing period until a steady state was again apparently obtained. The experimental procedure and the voltage application are illustrated in Figure 2. 


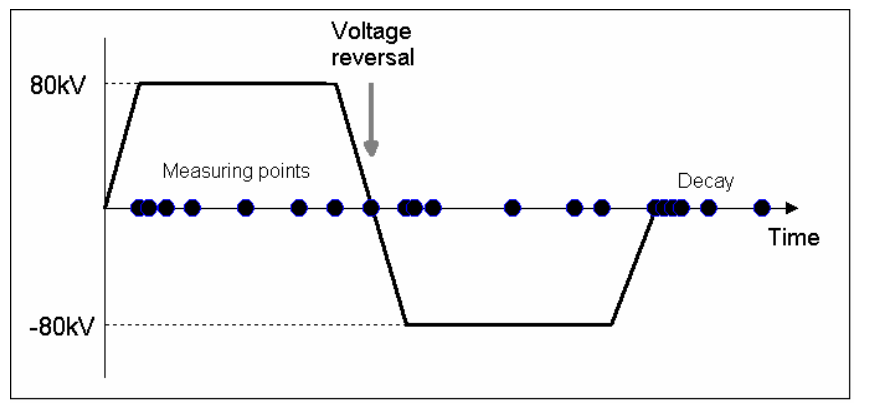

Figure 2. Voltage application procedure

\subsection{DATA PROCESSING AND CHARGE DENSITY CALIBRATION}

\subsubsection{GEOMETRICAL FACTOR AND CORRECTION}

PEA measurements made on plaque samples can assume that a uniform pulsed electric stress is applied and that the acoustic wave propagation is without divergence throughout the sample's thickness. This does not apply to cable geometry where the electric stress $e_{p}$ of the external pulse voltage, $v_{p}(t)$, is given by:

$e_{p}(t, r)=\frac{v_{p}(t)}{r \ln (b / a)}$

with $a$ and $b$ being the inner and outer radii of the insulation respectively. The PEA principle is based on the measurement of the acoustic wave emitted as a result of the interaction of the pulsed electric field and the space charge layer, with the delay in reception by the transducer defining the spatial location of the space charge emitting the pressure wave. The divergence of the pulsed electric field thus makes the intensity of the acoustic pressure wave initiated dependent not only on the charge density but also on its position.

It is generally assumed that the length of the cable sample is much greater than the insulation thickness and the material along the axial direction is homogeneous. The space charge distribution in the coaxial geometry therefore only varies in the radial direction and hence it can be concluded that the acoustic pressure wave representing the space charge density is also a function of the radial position. The pressure wave per unit area at position $r$ may be expressed as [19],

$$
p(t, r)=\gamma \frac{\partial \phi(t, r)}{\partial t}=-\frac{j u_{s a} A k}{\sqrt{r}} e^{j k\left(r-u_{s a} t\right)}
$$

where $\gamma$ is the density of the medium in which the acoustic wave is launched and travels through. This equation describes the propagation of an acoustic wave in an elastic (or lossless) medium in the radial direction within a cylindrical coordinate system. It is noticeable that the intensity of the pressure wave generated by the space charge layer inside the cable insulation decreases along the radial direction. This factor or ratio can be best described by

$$
\frac{p(t+\Delta t, b)}{p(t, r)}=(r / b)^{1 / 2}
$$

where $p(t, r)$ and $p(t+\Delta t, b)$ are the acoustic pressure wave intensities produced at radius $r$ and detected at the outer sheath $b$ after transmission through the insulation, $\Delta t$ is the time for the acoustic pulse travelling from position $r$ to $b$ (i.e. the position of outer semiconducting layer).

The divergent effects due to coaxial geometry have been taken into account for precise appraisal of the space charge distribution in cable samples by applying the geometry factor $(b / r)^{1 / 2}$ to the signal after the deconvolution, which is the result of combination of divergences of pulsed field and acoustic wave intensity in the radial direction [19].

\subsubsection{ATTENUATION AND DISPERSION COMPENSATION OF ACOUSTIC WAVE IN THICK INSULATION}

Apart from the divergence of the pulsed electric field and acoustic pressure wave in cylindrical geometry, the attenuation and dispersion caused by thick cable insulation to acoustic wave pressure is another negative aspect to the PEA technique which would introduce inaccuracy in the charge density measurement and loss of spatial resolution. However, this issue has been well discussed and a proper compensation (or recovery) algorithm has been derived and deployed in [19, 20] to give an accurate space charge distribution measurement, in particular, for thick samples. The reader is referred to $[19,20]$ for details of the derivation and the algorithm, which has been used here.

\subsubsection{CHARGE DENSITY CALIBRATION}

As the PEA technique is an indirect method of space charge profile measurement, the correlation between the acoustic wave and charge density needs to be determined by calibration. In the process of calibration a voltage is applied across the dielectric sample for a short period of time that is sufficiently low enough to ensure that no space charge is developed in the bulk sample, and only the capacitive charges on two electrodes are present. In the case of cable samples, for instance, the capacitive charge density induced at the inner and outer electrodes is proportional to the field at the interfaces, i.e.

$$
\begin{aligned}
& \sigma(a)=\varepsilon_{0} \varepsilon_{r} E(a) \\
& \sigma(b)=\varepsilon_{0} \varepsilon_{r} E(b)
\end{aligned}
$$

where $\varepsilon_{0}$ is the permittivity of free space, $\varepsilon_{r}$ the relative permittivity of the insulating material, $E(a)$ and $E(b)$ the electric stresses at the two interfaces respectively. Knowing the actual charge density at a given interface, the constant of proportionality between the output of the PEA system and charge density can be determined.

There has been a long-term interest in the determination of the electric field distribution in dc cables. This is governed by the conductivity of the insulating material. However the conductivity is determined by temperature and the electric stress, which it also determines. It is therefore difficult to predict the electric field distribution throughout the cable insulation under de voltage with the accuracy of that under ac voltage. The method used in the calculation of interface 
electric stress for charge density calibration was fully explained in [19], where the electric stress at a distance $r$ from the centre of the conductor was shown to be given by,

$$
E(r)=\frac{\delta r^{(\delta-1)} U}{b^{\delta}-a^{\delta}}
$$

where $\delta$ is a constant that is determined by the material and the temperature difference across the cable insulation. $U$ is the applied voltage. A value of $2 / 3$ is given for the constant $\delta$ in [21] and [22, 23], whereas a value of $1 / 2$ is quoted in [24] for XLPE without a temperature gradient. In view of the complexity of the problem, the value of $1 / 2$ for $\delta$ suggested in [24] has been adopted in this paper for the calculation of the electric stress needed for the purpose of charge density calibration.

\section{EXPERIMENTAL RESULTS 3.1 SPACE CHARGE DEVELOPMENT UNDER VOLTAGE POLARITY REVERSAL \\ 3.1.1 SPACE CHARGE ACCUMULATION WITH POSITIVE VOLTAGE AT CENTRAL CONDUCTOR}

Figure $3 \mathrm{a}$ illustrates the space charge evolution in the insulation of cable A when a positive voltage of $+80 \mathrm{kV}$ was applied to the central conductor. It was found that heterocharge gradually accumulated in the vicinities of the inner and outer electrodes and approached an apparently stable distribution within 90 minutes.

\subsubsection{SPACE CHARGE EVOLUTION FOLLOWING POLARITY REVERSAL AT THE INNER CONDUCTOR}

Following the measurements with positive voltage at the central conductor the external voltage polarity was switched to a negative polarity and space charge measurements again carried out. The results obtained during the polarity reversal (e.g. ramping up of the negative voltage on the inner conductor) are presented in Figure $3 \mathrm{~b}$. The heterocharge previously accumulated remains almost the same during the voltage ramp. See, for example, the positive charge labelled by $\boldsymbol{b}$ adjacent to the outer electrode. The continued existence

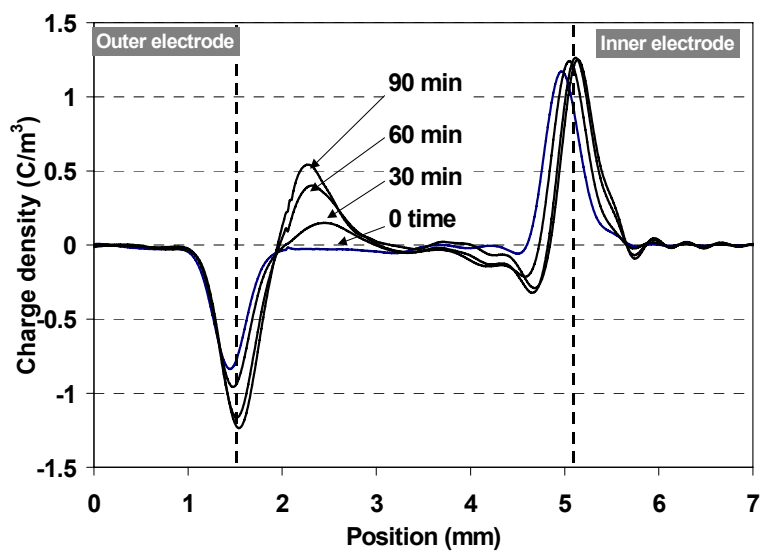

(a) Space charge accumulation with ageing time $(+80 \mathrm{kV}$ at central conductor) of this positive charge in the bulk insulation contributes a negative image charge on the outer electrode (peak $\boldsymbol{a}$ ) in addition to the positive capacitive charge from the applied voltage. Consequently the net electrode charge only has a small positive magnitude at this stage, albeit one that increases linearly with the applied voltage.

After the applied voltage reached its assigned reversal value of $-80 \mathrm{kV}$, the bulk space charge regions were first reduced in magnitude and then reversed polarity to become heterocharge again, as shown in Figure 3c. The polarity reversal occurs by the penetration of space charge of opposite polarity to the retained charge, which first reduces the magnitude of the retained charge and then cancels it out to form the reversed polarity peak in essentially the same position. This can be seen clearly in the space charge distribution in Figure 3c corresponding to 30 minutes at $-80 \mathrm{kV}$, which exhibits successive positive and negative peaks as one moves away from the outer electrode towards the inner conductor (anode). The final result of the cancellation is the formation of a new negative heterocharge peak, and a corresponding increase of the capacitive charge at the outer electrode/insulation interface. The time required for the space charge distribution to approach a new steady state is about 90 minutes just as was the case when the applied voltage had a positive polarity. The interesting point here is that this time is the same even though the negative heterocharge now accumulates in a region where positive space charge had been present as a result of the previous poling, whereas the data in Figure $3 \mathrm{a}$ was obtained for a sample that initially contained no space charge.

The steady state heterocharge distributions under reversed voltage polarity have almost the same shapes but the opposite polarities to those obtained initially. They even have the same charge accumulation rate. This phenomenon had been reported in $[16,25]$ and was termed the "mirror image effect" charge in [25]. Such "mirror image" charge distributions are clearly displayed in Figure 3d where the capacitive charge on both electrodes due to the external voltage has been removed.

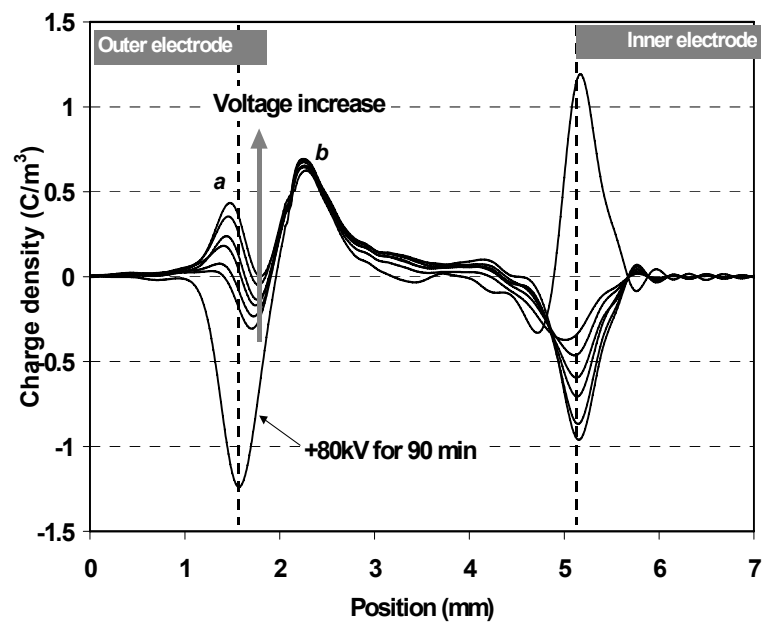

(b) Space charge during the polarity reversal ramp-up 


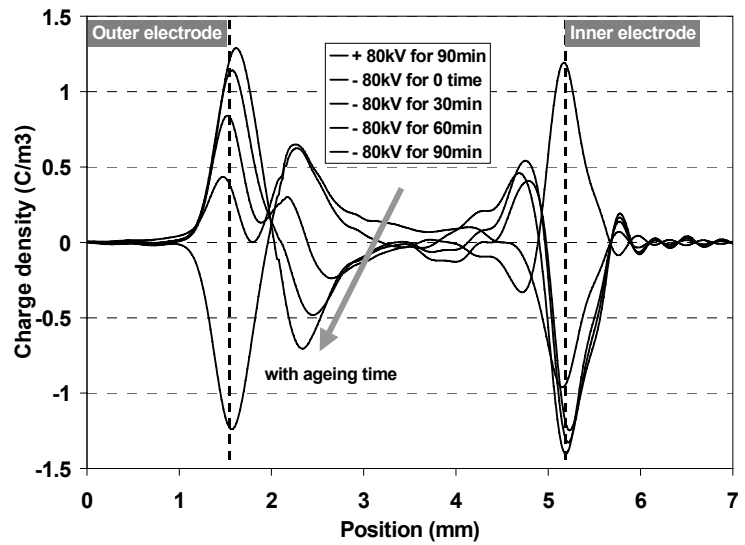

(c) Space charge accumulation with reversed voltage application time

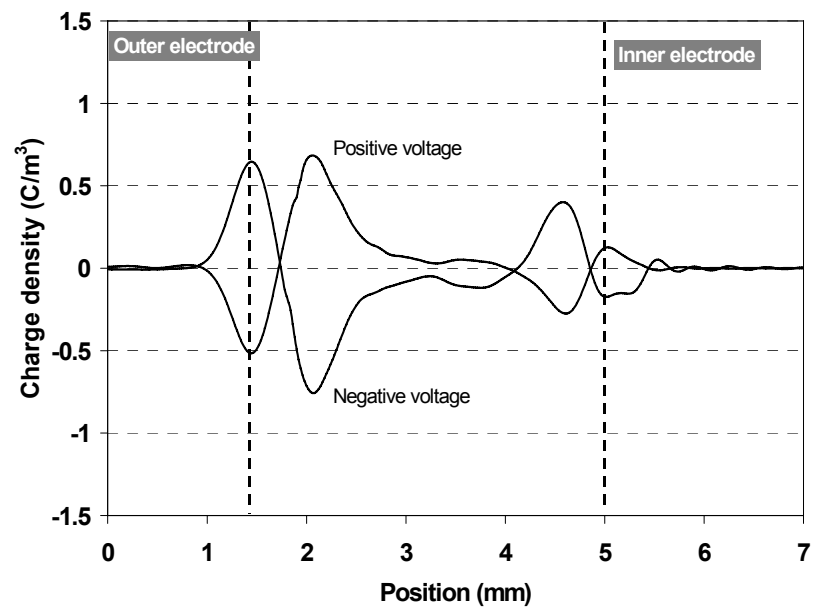

(d) "Mirror image" space charge distribution with capacitive charge removed from the electrode signal

Figure 3. Space charge development over the voltage polarity reversal

\subsection{SPACE CHARGE ACCUMULATION IN XLPE CABLES WITH A TEMPERATURE GRADIENT}

Cable B (prototype HVDC power cable with $5.7 \mathrm{~mm}$ thick insulation) was used to investigate the influence of a temperature gradient on space charge behaviour. First however, the space charge behaviour was measured for a uniform temperature (room temperature $\mathrm{T} \sim 25^{\circ} \mathrm{C}$ ) under the application of an external voltage of $+80 \mathrm{kV}$ to the inner conductor. This cable exhibited homocharge at the inner electrode and heterocharge at the outer electrode, and so all the measurements were carried out both with the external voltage applied and also with the external voltage temporarily switched off during the measurement. This procedure allowed a clear separation of the homocharge from the capacitive charge on the inner electrode.

Figure $4 \mathrm{a}$ shows the space charge accumulation obtained with temporary removal of the external voltage. The strongest feature is a heterocharge peak next to the outer electrode. A barely noticeable homocharge peak was also generated adjacent to the inner electrode (anode). After reaching a steady state distribution the external voltage was removed and the electrodes short-circuited, and measurements made of the space charge over a period of de-polarization. It was found that there was hardly any detectable change in the space charge distribution over 48 hours of de-polarization, see Figure 4.

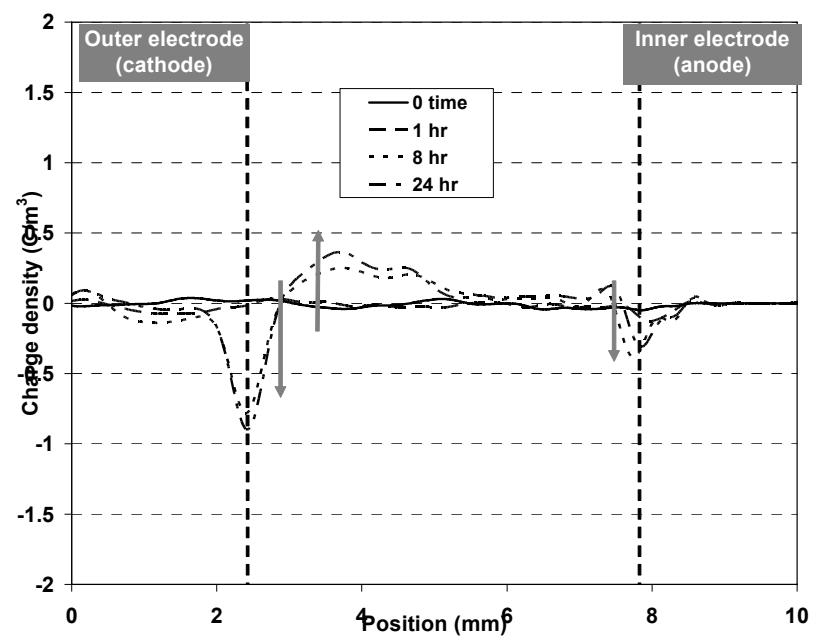

(a) Space charge accumulation under applied voltage (measurement with external voltage temporarily removed)

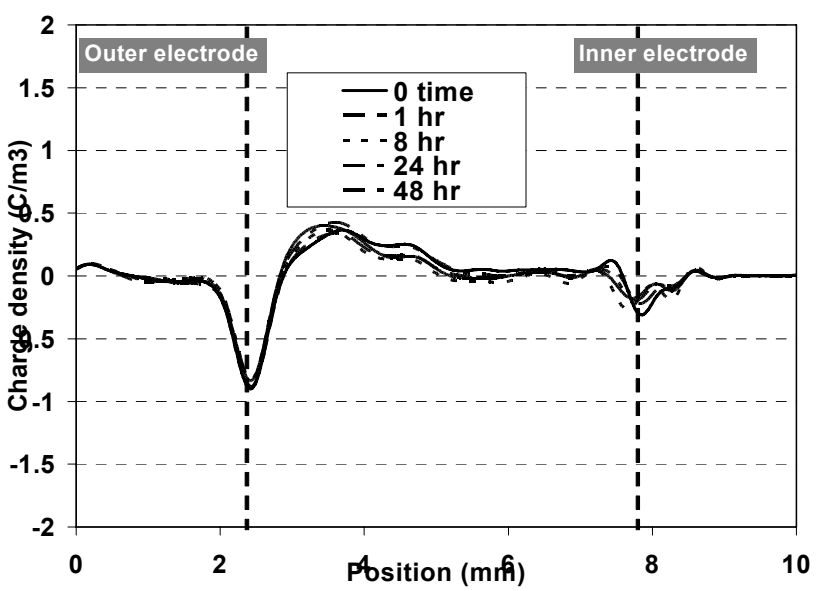

(b) Space charge profiles in decay after short-circuiting

Figure 4. Space charge behaviour in prototype HVDC cable B at room temperature

Measurements of space charge accumulation and decay in Cable B under a temperature gradient were carried out on a new piece of HVDC prototype cable. A temperature difference of $13{ }^{\circ} \mathrm{C}$ between conductor and outer sheath was obtained with the central conductor heated up to $70{ }^{\circ} \mathrm{C}$ while leaving outer semiconductor sheath in a natural convection condition. The space charge accumulation during polarization is shown in Figure 5a, and its subsequent behaviour during depolarization in Figure 5b. Because the space charge density in the bulk insulation is very small in comparison with that at a uniform room temperature its presence could only be discerned from the two induced image charge peaks at the outer and inner electrodes, obtained when the applied voltage was removed for measurement (i.e. the capacitive charge present during polarization was temporarily removed) . A very small positive charge peak (homocharge) was observed next to inner conductor (high temperature side), similar to that found at a uniform room temperature. In this case however, there is no measurable heterocharge near the outer conductor. A further difference is that the space charge accumulation 
reaches its saturation level within about two hours and then this amount of charge disappears within two hours following the removal of the applied voltage with the two electrodes short-circuited. In contrast room temperature accumulation takes about $24 \mathrm{~h}$ and the subsequent charge removal has not been achieved after $48 \mathrm{~h}$.

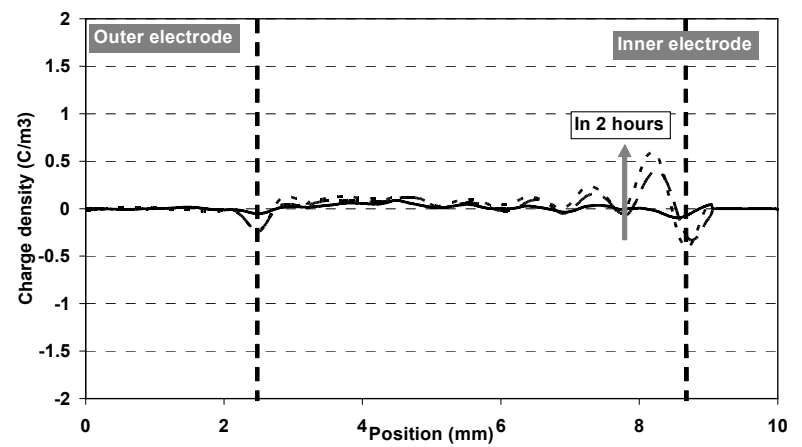

(a) Space charge accumulation (measured with external voltage temporarily removed)

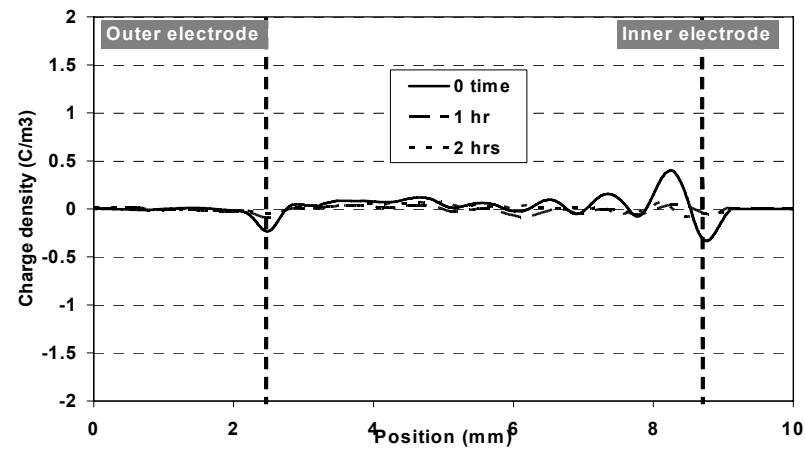

(b) Space charge decay after short-circuiting

Figure 5. Space charge behaviour in prototype HVDC cable B with temperature gradient

\section{DISCUSSION}

\subsection{ELECTRICAL FIELD AND SPACE CHARGE DISTRIBUTION DURING POLARIZARION AND VOLTAGE REVERSAL}

From a design engineers point of view the important factor during polarity reversal of a dc power cable is the behaviour of the electric field distribution. However this is both dependent upon and involved in the time dependence of the space charge. Therefore the transient field and space charge distribution must be discussed together.

Figure 6 shows the electric stress distributions calculated on the basis of the space charge profiles presented in figure 3 . During the 90 minutes of positive polarization $(+80 \mathrm{kV}$ on the central conductor) heterocharge at both electrode have increased the interfacial stresses at the outer and inner interface from the applied values of $20.5 \mathrm{kV} / \mathrm{mm}$ and 25.3 $\mathrm{kV} / \mathrm{mm}$ to $30.3 \mathrm{kV} / \mathrm{mm}$ and $27.3 \mathrm{kV} / \mathrm{mm}$ respectively. The electric stress in the central part of the insulation is reduced to a certain extent.

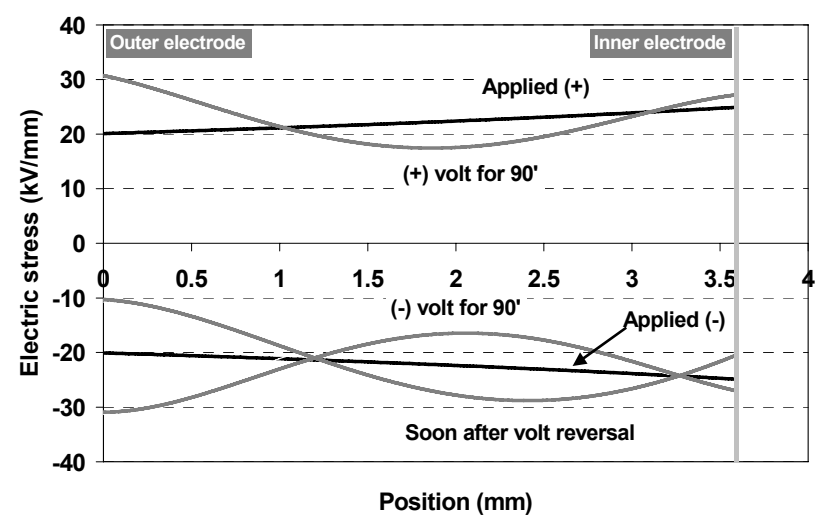

Figure 6. Electric stress distributions on voltage reversal

The heterocharge accumulation may be due to the separation of ionised species. This would be expected to give equal amounts of heterocharge at both electrodes in the absence of charge injection from the electrodes, since the electrode interfaces can be expected to be blocking for ionic species. However this is not the case here as there is a greater increase of the outer interface stress due to a larger amount of heterocharge in that region. This can be seen from Figure 3, where the charge density in the region of the inner electrode is smaller than that near to the outer electrode in both polarities. Since the volume of material is smaller for a given radial distance is smaller near the inner electrode it is clear that the amount of charge there is smaller than that near the outer electrode. It therefore seems that there is charge injection from the electrodes and that this is greatest at the inner conductor. However it has recently been shown [26] that heterocharge may be formed in cables by means of many very fast small charge packets injected from both electrodes. This would also explain the results obtained as long as injection from the inner electrode is greater than from the outer electrode.

The charge accumulated during polarization retains almost the same distribution during the voltage reversal ramp. The only changes during this period are the penetration of the space charge near the outer electrode into the bulk and reduction of the amount of space charge at the inner electrode. This latter feature tends to confirm the existence of charge injection from the inner conductor.

The electric stress in the central part of the insulation shows a significant enhancement. The maximum stress in this region is about $-30 \mathrm{kV} / \mathrm{mm}$, which is much higher than the applied value of $-25.3 \mathrm{kV} / \mathrm{mm}$ at the inner interface in the absence of space charge. On the other hand, the stresses at the inner and the outer interfaces are reduced to about $20 \mathrm{kV} / \mathrm{mm}$ and $10 \mathrm{kV} / \mathrm{mm}$ respectively, where the charge generated on polarization now becomes homocharge. As can be seen in Figure $3 \mathrm{c}$ the charge that reverses the polarity of the original space charge regions seems to originate from charge separation in the middle of the insulation where the field is largest. For a period of time both the original charge and the polarity reversing charge co-exist in the bulk, before the charge peaks near the electrodes change polarity. This can be seen from Figure 7, which shows the time-dependent 
behaviour of the integrated bulk charge density magnitude (i.e. total charge independent of polarity) for a one metre

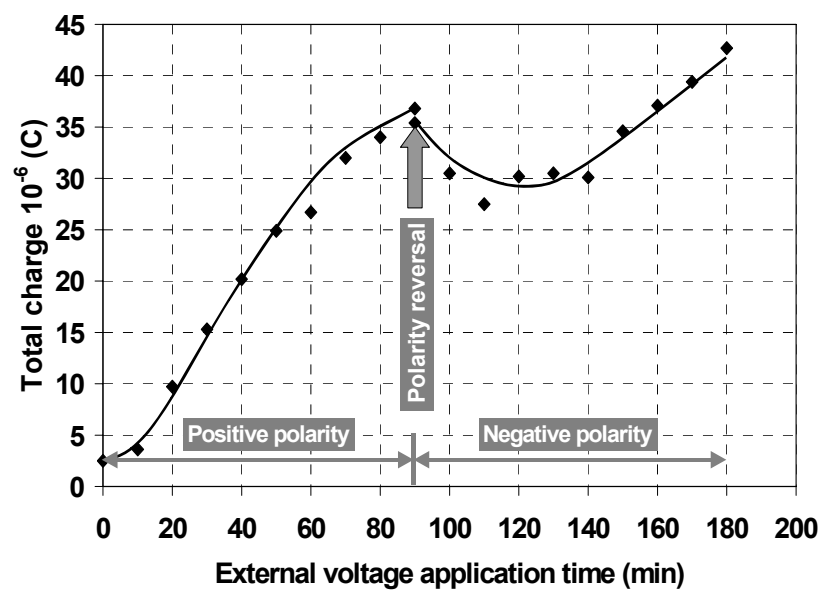

Figure 7. Total bulk charge magnitude through polarization cycle.

section of cable, throughout the polarization and polarity reversal cycle. The capacitive charge is excluded from the integration. It can be seen from Figure 7 that the total space charge in the cable section first decreases following polarity reversal, but then starts to increase without going through zero. Thus it is clear that the space charge regions produced during polarization do not just decrease, pass through zero, and then reverse polarity. Instead compensating space charge regions co-exist with the original space charge. The observation in Figure $3 \mathrm{c}$ that these compensating charges originate in the middle of the cable correlates their generation with the electric field maximum that exists there when the polarity reversal is made.

These results show that the dynamics of space charge accumulation on polarity reversal is not a time reversal image of the dynamics that occur during polarization. The field distribution as modified by the existing space charge has an effect on the space charge dynamics, and charge generation may not take place in the same location as it did on initial polarization This result suggests the way in which polarity reversal may influence insulation reliability. Space charge generation in the middle of the insulation and its movement towards the electrode regions will cause two high field regions to move towards the electrodes with increasing local currents and possibly charge recombination in the electrode/interface regions, both of which may cause damage [27, 28].

Figure 7 also shows that the space charge distribution has not reached a steady state after 90 minutes of polarization, though it is showing signs of saturation. In addition it can be seen that the total charge on polarity reversal is higher than that obtaining on initial polarization. An inspection of figure $3 \mathrm{~d}$ shows that this is because the amount of heterocharge at the inner electrode is greater than it was during initial polarization, i.e. an exact mirror image has not been formed during the poling time. Nonetheless the electric field distribution obtaining after 90 minutes of re-polarization at $80 \mathrm{kV}$ is almost a mirror image of that found after 90 minutes of polarization at $+80 \mathrm{kV}$.

\section{2 "MIRROR IMAGE EFFECT" CHARGE DISTRIBUTION}

The mirror image effect [25] refers to steady state space charge distributions that are inverses of one another when the cable conductor is subjected to voltages of opposite polarity. This effect occurs whether the opposite polarity was achieved by polarity reversal as here, or by the application of opposite polarity voltages to different sections of the same cable [29]. An example of the latter feature measured on a different cable [29] is given in figure 8. This figure also shows that the mirror effect includes space charge of both polarity at the outer electrode. The injected charge can be seen as an extension of the capacitive peak into the insulation over a period of time, denoted as 'HOMOCHARGE' on the Figure. Clearly therefore the mirror effect occurs regardless of the nature and origin of the space charge.

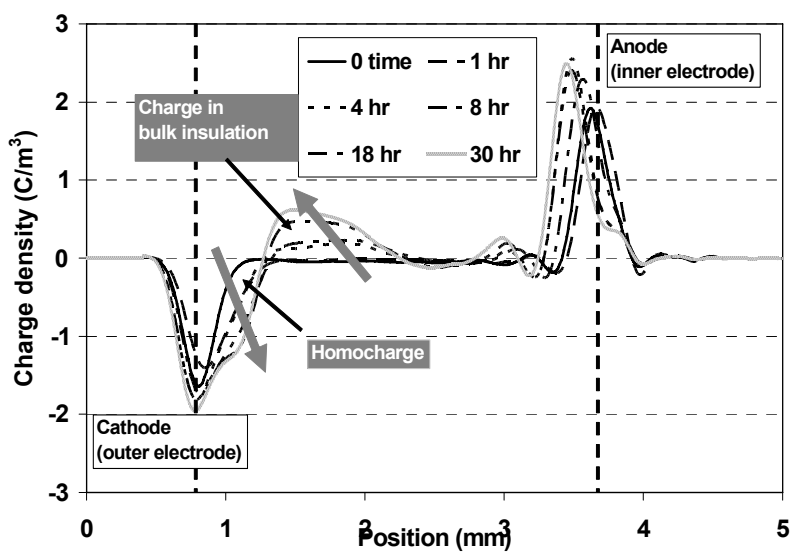

(a) Positive voltage at central conductor

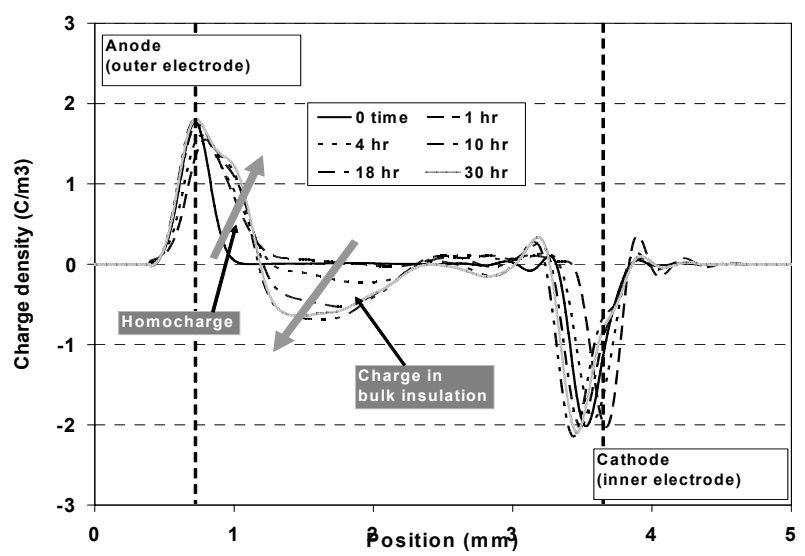

(b) Negative voltage at central conductor

Figure 8. Space charge distributions in a third type of XLPE cables with opposite voltages applied at central conductor. Taken from [29].

Our results have also shown that the mirror effect is not preserved during the charge dynamics brought about by polarity reversal. The mirror image charge effect is thus a steady state feature independent of the nature of the space charges, and the way that their distribution is produced. Since the key feature defining a steady state is that the radial current must be the same at all radial position it must be concluded that the field distribution produced by the mirror space charge distribution is one such that current is everywhere the same, 
including the currents across the electrode interfaces. The different values of the interface fields resulting from differences in space charge magnitude reflect the differences in geometry and nature of the respective electrodes. A mirror image effect in the space charge and electric field distributions will be produced when an opposite polarity voltage is applied to the conductor if and only if the current across each of the electrode interfaces has the same value for the same voltage magnitude, whatever the voltage polarity. This is the essential meaning of the mirror charge effect [25]. An explanation for the mirror charge effect therefore requires an explanation as to why the electrode interface current magnitude should only depend upon the electric field magnitude and not its polarity. This implies that there is no contact charge layer formed in the insulator, as the contact charge field would introduce an asymmetry between positive and negative cross-interface currents. This means that there is no difference between the chemical potential (Fermi energy) of an electron in the electrode material and in the XLPE at the interface. Such a situation is likely for the carbon loaded polyethylene semiconductor electrodes used in cables. An understanding of the process whereby charge carriers pass from the electrode into the insulator is required to complete the explanation, and this is not yet available. Two alternative mechanisms have been proposed: thermal activation via the vacuum state [30] to and from states within the polymer, and tunnelling, with and without thermal assistance, between the Fermi energy of the electrode and donor and acceptor states within the polymer located in a narrow region of energy around the Fermi energy of the polymer at the interface [31]. Of the two, the tunnelling process [31] seems the most likely in this case as the energies of the states between which the transfer occurs will be very close and any thermal assistance will be to the most effective state for tunnelling This intermediate state will be the same whether electrons are being transferred from the electrode or to the electrode. The interface electric field will control the current in this case via its influence upon the height and more particularly the width of the interface barrier (see section 9.2.2. of [32]).

The origin of the space charge is irrelevant to the generation of the mirror image effect however the generation of compensating charges in the middle of the insulation during polarity reversal in cable A indicates that in that case they are likely to be field-separable ionic species [33-35]. In particular the lack of a change in the positive charge around the outer electrode during the polarity reversal ramp up indicates that the positive charge has a low mobility and that the mobile species is the negative charge. What is clear though is that the conductivity of mobile charge species in the insulating material is greater than the conductivity of charges transferring across the electrode interfaces before the space charge distribution reaches a steady state. It is this that leads to the formation of hetero space charge regions, which increase the interface fields and reduce the bulk field so that the current across the interface can come into coincidence with the bulk current. On polarity reversal the reduction of the interface fields reduces the interface currents, while the high field in the middle process the space charge currents that invert the charges at the interfaces.

\subsection{SPACE CHARGE ACCUMULATION AT ELEVATED TEMPERATURE}

Cable B was used for the measurements in elevated temperature and so the results are not strictly comparable with those of cable A. However the space charge distribution at a uniform temperature of $\sim 25{ }^{\circ} \mathrm{C}$ approaches a steady state that is very similar to that found in cable A, Figure $3 \mathrm{a}$, with a large positive heterocharge at the outer electrode when the conductor had a potential of $+80 \mathrm{kV}$. The difference is that the heterocharge peak is spread more widely than in cable $\mathrm{A}$, and there is a small amount of homocharge at the inner conductor that is reducing with time. The time taken to reach the steady state is much longer than in cable A, possibly because the insulation thickness is larger so that the charge transit time is longer. The longer transit time may also be the reason why a negative heterocharge has not yet appeared near the inner electrode. The positive charge at the outer conductor shows very little evidence of decaying when the cable was short-circuited just as the positive heterocharge in cable A did not reduce during the polarity reversal ramp up, see Figure $3 \mathrm{~b}$. It therefore appears that this charge is the same in both cables, i.e. a relatively immobile ion species originating with the volatile cross-linking by products that are present in both cables. The measurements made in a temperature gradient with a mean elevated temperature can therefore be used to gain some insight into the effect of temperature on space charge distributions., and hence indirectly upon the temperature dependence of the bulk and interface currents. Figure 5a shows that higher temperatures has removed the heterocharge region near to the outer electrode and increased the homocharge at the inner electrode. This behaviour is consistent with a situation in which the interface currents have increased more rapidly with the increase in temperature than the current in the body of the insulator, i.e. the interface currents are activated with higher activation energy than the currents in the insulation material. The inner electrode with a higher temperature than the bulk material and the outer electrode, and thus has a higher injection current than the bulk current and so gives a net homocharge, which reduces the interface field at the inner electrode (to $\sim 17 \mathrm{kV} / \mathrm{mm}$ ) to bring the two into coincidence. The temperature at the outer electrode is less than that in the bulk material but its increase is still sufficient to increase the interface current there and bring it into coincidence with the bulk current without a significant build-up of space charge. Similar results have been found recently for MVdc-cables at a similar applied field [36] (i.e. $10 \mathrm{kV} / \mathrm{mm}$ in [36], here $\sim 14 \mathrm{kV} / \mathrm{mm}$ ), and temperature gradient close to that obtaining here. In view of our speculation that the injection current involved carrier tunnelling through a potential barrier this result shows that the optimum process involves thermal activation from around the relevant Fermi energy to a state where the barrier width is sufficiently reduced to allow tunnelling to become effective [31, 37].

While it clear that in these measurements the elevated temperature has almost eliminated space charge in the cable, this cannot be taken as an axiom. Different interfaces and bulk material may have different conductivity activation energies 
and may result in an inversion of the steady state space charge to homocharge. In this case polarity reversal will result in transient high interface fields, and hence the reversing charges will be produced by interface currents. This may be more damaging to the cable than charge generation in the middle of the cable by ion separation.

\section{CONCLUSIONS}

It has been shown that the mirror image charge effect is a steady state effect that is independent of the nature and type of the charges and the dynamics involved in the formation of the charge distribution. The reason for the occurrence of a mirror space charge distribution is that the currents across the electrode interface are dependent only upon the magnitude of the interface field and not its polarity. It is suggested that these currents are produced by a thermally assisted tunnelling of charge carriers through the interface barrier.

Measurements made during the polarity reversal process show that the space charge reversal is influenced by the preexisting field distribution. Consequently space charge is generated in the middle of the insulation, where the field is higher than the design stress. This leads to currents moving charge from the bulk to the interfaces, and a high field position moving towards the electrodes that may be a source of damage.

Temperature gradients such as will be found in service change the space charge distribution because the activation energies for the cross-interface currents are different to those for bulk transport. Here the space charge is much reduced, but this may not always be the case as the outcome depends upon details of the interface and semiconductor material among other things.

\section{REFERENCES}

[1] Y. Zhang, J. Lewiner, C. Alquie, and N. Hampton, "Evidence of strong correlation between space-charge buildup and breakdown in cable insulation", IEEE Trans. Dielectr. Electr. Insul., Vol. 3, pp.778$783,1996$.

[2] N. Hozumi, H. Suzuki, T. Okamoto, K. Watanabe and A. Watanabe, "Direct Observation of Time-dependent Space Charge Profiles in XLPE Cable under High Electric Fields", IEEE Trans. Dielectr. Electr. Insul., Vol. 1, pp. 1068-1076, 1994.

[3] R. Bartnikas, "Performance characteristics of dielectrics in the presence of space charge", Trans. Dielectr. Electr. Insul., Vol. 4, pp. 544-557, 1997.

[4] P. K. Watson, "The transport and trapping of electrons in polymers", IEEE Trans. Dielectr. Electr. Insul., Vol. 2, pp. 915-924, 1995.

[5] B. Sanden, "Space charge accumulation in fresh and degassed XLPE insulation", 28th Dielectr. Soc. Meeting, UK, pp.225-233, 1998.

[6] K. R. Bambery and R. J. Fleming, "The temperature dependence of space charge accumulation in cross-linked polyethylene", J. Thermal Analysis, Vol. 50, pp. 19-31, 1997.

[7] Y. Tanaka, G. Chen, Y. Zhao, A. E. Davies, A. S. Vaughan and T. Takada, "Effect of additive on Morphology and space charge accumulation in low density polyethylene", IEEE Trans. Dielectr. Electr. Insul., Vol. 10, pp. 148-154, 2003.

[8] L. A. Dissado, G. Mazzanti and G. C. Montanari, "The role of trapped space charges in the electrical aging of insulating materials", IEEE Trans. Dielectr. Electr. Insul., Vol. 4, pp. 496-506, 1997.

[9] A. See, L. A. Dissado and J. C. Fothergill, "Electric field requirements for charge packet generation and movement in XLPE", IEEE $7^{\text {th }}$ Intern. Conf. Solid Dielectrics, pp.232-235, Eindhoven, the Netherlands, 2001.
[10] M. Mammeri, C. Laurent and J. Salon, "Influence of space charge build up on the transition to electrical treeing in PE under ac voltage", IEEE Trans. Dielectr. Electr. Insul., Vol. 2, pp. 27-35, 1995.

[11] G. Chen, T. Y. Tay, A. E. Davies, Y. Tanaka and Takada, "Electrodes and charge injection in low-density polyethylene using the pulsed electroacoustic technique", IEEE Trans. Dielectr. Electr. Insul., Vol. 8, pp. 867-873, 2001.

[12] T. Maneno, T. Futami, H. Kushibe, T. Takada and C. M. Cooke, "Measurement of Spatial Charge Distribution in Thick Dielectrics Using the Pulsed Electroacoustic Method", IEEE Trans. Electr. Insul., Vol. 23, pp. 433-439, 1988.

[13] K. Fukunaga, H. Miyata, T. Takhaashi, S. Yashia and T. Niwa, "Measurement of Space Charge Distribution in Cable Insulation Using the Pulsed Electroacoustic Method", Pro. $3^{\text {rd }}$ Int. Conf. on Polymer Insulated Power cables, Versailles, France, pp. 520-525, 1991.

[14] R. Liu, T. Takada and N. Takasu, "Pulsed Electroacoustic Method for Measurement of Charge Distribution in Power Cables under both AC and DC Electric Field”, J. Phys. D. Appl. Phys, Vol. 26, pp. 986-993, 1993.

[15] N. Hozumi, H. Suzuki, T. Okamoto, K. Watanabe and A. Watanabe, "Direct Observation of Time-dependent Space Charge Profiles in XLPE Cable under High Electric Fields, IEEE Trans. Dielectr. Electr. Insul., Vol. 1, pp. 1068-1076, 1994.

[16] X. Wang, D. Tu, Y. Tanaka, T. Muronaka, T. Takada, C. Shinoda and T. Hashizumi, "Space charge in XLPE power cable under dc electrical stress and heat treatment", IEEE Trans. Dielectr. Electr. Insul., Vol. 2, pp. 467-474, 1995.

[17] T. Mureonaka, Y. Tanak, T. Takada, S. Maruyama and H. Mutou, "Measurement of space charge distribution in XLPE cable using PEA system with flat electrode", IEEE Conf. Electr. Insul. Dielectr. Phenomena (CEIDP), San Francisco, USA, pp. 266-269, 1996.

[18] T. Tanaka and A. Greenwood, Advanced power cable technology, Volume I, CRC Press Inc., USA, pp. 144-145, 1983.

[19] M. Fu and G, Chen, "Space Charge Measurement in Polymer Insulated Power Cables Using Flat Ground Electrode PEA System", IEE Proc. Sci. Measur. Technol. Vol. 150, pp.89-96, 2003.

[20] Y. Li, M. Aihara, K. Murata, Y. Tanaka and T. Takada, "Space charge measurement in thick dielectric materials by pulsed Electroacoustic method", Rev. Sci. Instrum., Vol. 66, pp. 3909-3816, 1995.

[21] Z. Liu, Electrical Insulation Design, - Power Cables, Mechanic Industry Press, Beijing, China (in Chinese), 1981.

[22] I. W. McAllister, G. C. Chichton and A. Pedersen, "Charge Accumualtion in DC Cable: a Macroscopic Approach", IEEE Int. Symp. Electrical Insulation (ISEI), Pittsburgh, PA, USA, pp. 212-216, 1994.

[23] I. W. McAllister, G. C. Chichton and A. Pedersen, "Space Charge Field in DC Cables", IEEE Int. Symp. Electrical Insulation (ISEI), Montreal, Canada, pp. 661-665, 1996.

[24] T. Tanaka and A. Greenwood, Advanced power cable technology, Volume I, CRC Press Inc., USA, 1983.

[25] K. R. Bambery and R. J. Fleming, "Space charge accumulation in two cable grades of XLPE", IEEE Trans. Dielectr. Electr. Insul., Vol. 5, pp. 103-109, 1998.

[26] S. Delphino, D. Fabiani, G.C. Montanari, L.A. Dissado, C. Laurent and G. Teyssedre, "Fast charge packet dynamics in XLPE insulated cable models", IEEE Conf. Electr. Insul. Dielectr. Phenomena (CEIDP), (IEEE pub.07CH37929), pp. 421-424, 2007.

[27] J. Artbauer, "Electric strength of polymers", J. Phys. D: Appl. Phys, Vol. 29, pp. 446-456, 1996

[28] C. Laurent, "Optical pre-breakdown warnings in insulating polymers", IEEE Electr. Insul. Mag., Vol. 15, No. 2, pp. 5-13, 1999.

[29] M. Fu, G. Chen and J. C. Fothergill, "Mirror image effect space charge distribution in XLPE power cable under opposite stressing voltage polarity", Proc. Of $14^{\text {th }}$ Inter. Symp. on High Voltage Eng. (ISH), Beijing, China, 2005 (paper H-03, CD-ROM).

[30] D.K. Davies, "Charge generation on dielectric surfaces", Brit. J. Appl. Phys . (J. Phys. D), ser.2, Vol.2, pp. 1533-1537, 1969.

[31] T. J. Lewis, "Electrical effects at interfaces and surfaces", IEEE Trans. Electr. Insul., Vol. 21, pp. 289-295, 1986.

[32] L.A. Dissado and J.C. Fothergill, Electrical Degradation and Breakdown in Polymers (P.Peregrinus Ltd for IEE, London, 1992)

[33] T. Takada, "Space charge formation in dielectrics", IEEE Trans. Electr. Insul., Vol. 21, pp. 873-877, 1986. 
[34] M. Ieda, "Carrier injection, space charge and electrical breakdown in insulating polymers", IEEE Trans. Electr. Insul., Vol. 22, pp. 261-267, 1987.

[35] K. S. Suh, S. J. Hwang, J. S. Noh and T. Takada, "Effect of constituents of XLPE on formation of space charge", IEEE Trans. Dielectr. Electr. Insul., Vol. 1, pp. 1077-1083, 1994.

[36] D. Fabiani, G.C. Montanari, C. Laurent, G. Teyssedre, P.H.F. Morshuis, R. Bodega, and L.A. Dissado, "HVDC cable design and space charge accumulation: Part 3 effect of temperature gradient", IEEE Elect. Insu. Mag., to be published, 2008.

[37] R. M. Hill and L.A. Dissado, "The temperature dependence of relaxation processes", J. Phys. C: Solid State Phys., Vol. 15, pp. 5171$5193,1982$.

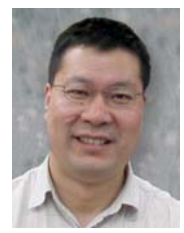

Mingli Fu was born in Shannxi, China in 1962. He received the B.Eng. degree in electrical engineering from Xian Jiaotong University, China in 1983. He spent many years at China Electric Power Research Institute (CEPRI) as a Research and Development engineer before he came to UK as a visiting research fellow at the University of Southampton in 1997, and later on obtained the Ph.D. degree from the same university. He is currently a lecturer at the University of Leicester, UK. His research interests lie in dielectric materials, high voltage technology and the insulation system ageing and integrity diagnosis.

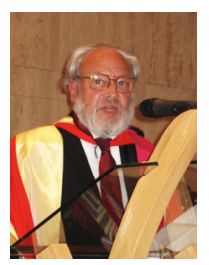

Len A. Dissado (SM'96-F'06) was born in: St. Helens, Lancashire, U.K on 29 August 1942. He was educated at Thomas Linacre Technical School, Wigan, Lancashire, 1953-1960, gaining a State Scholarship for University Entry in 1959. $\mathrm{He}$ graduated from University College London with a $1^{\text {st }}$ Class degree in Chemistry in 1963 and was awarded the Ph.D. degree in theoretical chemistry in 1966 and the D.Sc. degree in 1990. After rotating between Australia and England twice he settled in at Chelsea College in 1977 to carry out research into dielectrics. His interest in breakdown and associated topics started with a consultancy with STL begun in 1981. Since then he has published many papers and one book, together with John Fothergill, in this area. In 1995 he moved to The University of Leicester, and was promoted to Professor in 1998. He has been a visiting Professor at The University Pierre and Marie Curie in Paris, Paul Sabatier University in Toulouse, and Nagoya University, and has given numerous invited lectures, the most recent of which was the Whitehead lecture at IEEE Conference on Electrical Insulation and Dielectric Phenomena (CEIDP 2002) in Cancun, Mexico. He was awarded the title Docteur Honoris Causa by the Universite Paul Sabatier in 2007. Currently he is an Associate Editor of IEEE Transactions on Dielectrics and Electrical Insulation, co-chair of the Multifactor Aging Committee of DEIS and a member of DEIS Administrative Committee.

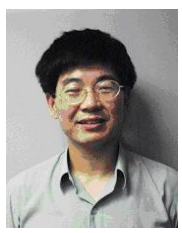

George Chen was born in China in 1961. He received the B.Eng. (1983) and M.Sc. (1986) degrees in electrical engineering from Xian Jiaotong University, China. After he obtained the Ph.D. (1990) degree from the University of Strathclyde, UK, on the work of permanent changes in electrical properties of irradiated low-density polyethylene, he joined the University of Southampton as postdoctoral research fellow and became senior research fellow subsequently. In 1997 he was appointed as a research lecture and promoted to a Reader in 2002. Over the years, he has developed a wide range of interests in high voltage engineering and electrical properties of materials and published over 100 papers.

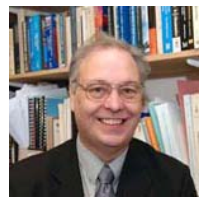

John C. Fothergill (SM'95-F'04) was born in Malta in 1953. He graduated from the University of Wales, Bangor, in 1975 with a Batchelor's degree in electronics. He continued at the same institution, working with Pethig and Lewis, gaining a Master's degree in electrical materials and devices in 1976 and doctorate in the electronic properties of biopolymers in 1979. Following this he worked as a senior research engineer leading research in electrical power cables at STL, Harlow, UK. In 1984 he moved to the University of Leicester as a lecturer. He now has a personal chair in engineering and is currently Pro-ViceChancellor. 